Pour les décès totaux, nous relevons, en 1902, un pourcentage de 11,3 par rapport aux naissances; ce pourcentage a atteint les extrêmes de 12,5 et de 6,8 , soit une moyenne de 10,2 , pour l'ensemble de la période trentenaire.

En ce qui concerne les décès par entérite et diarrhée par rapport aux naissances, on remarque pareillement qu'en 1902, le pourcentage était de 3,7 et en 1932 de 1,3. Ce dernier chiffre est le plus bas pourcentage de la période, le plus élevé étant de 4,1 en 1911.

Il faudrait, pour pouvoir tirer un enseignement de ces chiffres, les comparer à la période trentenaire précédente. Dans l'impossibilité où nous nous sommes trouvés pour pouvoir le faire, nous croyons judicieux de diviser la période 1902-1932, en deux périodes de quinze années. Ainsi, en ce qui concerne le pourcentage des décès totaux par rapport aux naissances, on trouvera pour la première période un pourcentage moyen de 10,6 ; dans la seconde période, on aura 9,2 , soit une amélioration moyenne voisine de $13 \%$.

En ce qui concerne le pourcentage des décès par entérite et diarrhée, on trouvera dans les mêmes conditions et en partant des mêmes bases, une moyenne de 3,1 pour la première période et de 2,2 pour la seconde, soit une amélioration d'environ $30 \%$.

C'est là, on le voit un résultat très notable.

Si l'on considère, malgré certaines tentatives actuelles d'alimentation différente, que le lait est le seul aliment des enfants en dessous de 1 an, que celui-ci en tant qu'aliment de remplacement est la cause déterminante des diarrhées et entérites mortelles, on doit convenir, sans pour cela méconnaître les autres facteurs de l'hygiène infantile, que les améliorations dans l'hygiène laitière se trouvent là largement illustrées.

\title{
LES SAVEURS ET ODEURS ANORMALES DU LAIT
}

par

\section{Jean PIEN}

Docteur ès-Sciences

Ingénieur chimiste (I.C.R.)

Directeur des Laboratoires des "Fermiers Réunis "

\section{et S. HERSCHDOERFER}

Docteur ès-Sciences

(Vienne)

\section{(Suite)}

\section{Etude du quatrième groupe de causes.}

Modification des éléments normalement contenus dans le lait par des facteurs d'ordre physico-chimique.

Les causes d'altération qui relèvent de cé groupe peuvent se réduire presque exclusivement à l'oxydation catalytique des matières grasses du lait. 
Ces oxydations peuvent être produites soit par des agents physiques, soit par des agents chimiques, soit par des agents diastasiques.

II est essentiel de faire immédiatement une distinction importante en ce qui concerne les altérations possibles de la matière grasse :

Il ressort des recherches de nombreux auteurs (WEIGMANN, Porcher, Hunziker) que les corps gras du lait peuvent subir :

$1^{\circ}$ Soit une hydrolyse (généralement diastasique) qui provoque un dédoublement en acides gras et glycérine ;

20 Soit une oxydation interne intéressant surtout les acides gras non saturés pouvant être l'œuvre de certaines diastases mais aussi de certains agents physiques ou chimiques agissant comme catalyseurs.

Dans le premier cas on assisterait à la haissance de la rancidité ; dans le second cas au "suiffage » ou à "l'huilage ".

Il faut noter, par conséquent, que la rancidité n'est pas une oxydation, mais une hydrolyse. Elle s'accompagne d'ailleurs quelquefois d'une oxydation ; c'est pourquoi on constate parfois le suiffage en même temps que la rancidité et cela explique que ces deux notions différentes aient pu être confondues.

Nous avons voulu grouper dans ce paragraphe ce qui concerne l'oxydation de la matière grasse par des agents physiques ou chimiques.

\section{OXYDATION SOUS L'INFLUENCE D'AGENTS PHYSIQUES}

Nous n'envisageons que le cas de la lumière solaire. - C'est un fait bien connu que la lumière solaire est capable d'activer l'oxydation des corps gras du lait et de provoquer le goût de suif caractéristique de ce phénomène.

WhitehEAD [26] a constaté que cette oxydation intéresse la matière grasse du lait puisque le lait écrémé ne la donne pas. Il a montré qu'elle est bien due à la lumière solaire puisque, toutes choses égales d'ailleurs, elle ne se produit pas à l'obscurité.

Comme, d'autre part, le lait chauffé à haute température donne le même résultat que le lait cru, on est fondé à admettre qu'il n'y a pas lieu ici de faire intervenir une action biologique. La lumière solaire agissant sur la matière grasse du lait (en l'absence de toute substance ou de tout facteur étranger au lait normal chauffé) est eapable de l'oxyder.

Le mécanisme de cette oxydation est done bien strictement physico-chimique.

Or, en même temps qu'on assiste à l'oxydation des graisses, on 
peut constater (même dans le lait chauffé) la réduction du bleu de méthylène.

WhiteheAD estime selon toute vraisemblance que le lait exposé au soleil est le siège de réactions oxydo-réductrices. Quelques substances du lait agissent comme récepteurs d'hydrogène au cours de l'oxydation de la graisse et un potentiel de réduction se développe dans le lait. Si on se trouve en présence de bleu de méthylène, celui-ci peut agir à son tour comme récepteur d'hydrogène au moment où on atteint une certaine valeur du potentiel de réduction et on assiste à sa décoloration - en l'absence bien entendu de tout microbe et de toute diastase.

Cette donnée, intéressante pour l'explication de l'oxydation catalytique par la lumière solaire, est très importante à un autre point de vue : il en découle en effet que l'épreuve au bleu de méthylène pour l'appréciation rapide de la qualité bactériologique du lait doit être effectuée à l'obscurité.

Notons encore que, pour Tracy [25], l'action de la lumière solaire peut donner une saveur de brûlé, intéressant vraisemblablement les composés non gras du lait à côté de l'altération suiffeuse qui concerne les matières grasses.

\section{OXYDATION SOUS L'INFLUENCE D'AGENTS CHIMIQUES}

\section{A. INFLUENCE DE L'OZONE.}

Il est facile de concevoir qu'en soumettant le lait, à l'action de l'air ozonisé on provoquera rapidement une oxydation dans la matière grasse. Nous avons personnellement développé un goût de suif très intense dans de la crème en $\mathrm{y}$ faisant barbotter de l'air faiblement ozonisé pendant quelques minutes. Ces phénomènes ne doivent pas être méconnus quand on se propose de faire l'irradiation du lait sans précautions spéciales. (Ils indiquent en outre qu'il faut s'interdire de désinfecter par l'ozone l'eau destinée au lavage du beurre et l'air des chambres frigorifiques où le beurre est stocké.)

\section{B. Influence des sels métalligues.}

De très nombreux auteurs ont étudié l'influence eatalysante de certains sels métalliques sur l'oxydation des graisses.

Disons tout de suite que le cuivre est le métal dont l'action est la plus redoutable. Il y a, à ce sujet, une parfaite unanimité dans tous les travaux relatifs à cette question.

a) Relations entre la présence d'appareils en cuivre et l'apparition de certaines saveurs anormales. - Pour GuTHRIE [27] il n'y a aucun doute que certaines saveurs huileuses, suiffeuses, "oxydées ", astringentes même, sont dues à la corrosion des métaux employés en laiterie. 
Les saveurs désagréables les plus importantes sont dues au cuivre et à des alliages d'abord, ensuite au cuivre chromé par électrolyse, au cuivre étamé (qui se đésétame si facilement), au nickel, à certains alliages à base de nickel ou à base de chrome. Toutefois l'alliage nickel-chrome sans autres métaux paraît excellent et ne communique aucune saveur. Il en est de même de l'aluminium qui ne présente aucun inconvénient à cet égard.

Pour W егсн [28] une grande partie des mauvais goûts rencontrés dans le lait et ses dérivés proviennent de leur séjour dans des appareils en cuivre ou de leur contact avec des parties d'appareils en laiton. Cet auteur note une rapide usure des appareils en laiton en contact avee le lait. L'augmentation de la teneur du lait en cuivre confirme cette donnée que nous préciserons tout à l'heure par des chiffres. Comme, d'autre part, le mauvais goût coïncidait avec l'introduction de parties en laiton dans les appareils, WEICH n'hésite pas, à la suite de nombreuses observations, à rendre le cuivre responsable des mauvais goûts constatés.

RICE [29] constate que le cuivre est directement responsable du suiffage du lait (lait condensé en particulier). Il apporte en outre les précisions suivantes : le lait prend plus de cuivre et plus de mauvais goût au contact de l'air que dans le vide. Mais il estime qu'il vaut encore mieux supprimer le cuivre que de travailler dans le vide. Un appareil de cuivre inutilisé pendant quelque temps provoque plus de mauvais goût (en abandonnant plus de métal) qu'un appareil qui sert constamment et qui est parfaitement poli et bien nettoyé, Cet auteur note, encore, que le lait qui passe dans un appareil en cuivre entraîne au début plus de cuivre et présente plus de mauvais goût que le lait qui passe ensuite. Le premier lait sorti de l'appareil est inutilisable et doit être employé à d'autres usages.

Observations analogues de la part de Matrick [30]. Mais cet auteur attache encore plus d'importance à l'influence de l'air. En l'absence d'oxygène, le euivre ne donnerait pas d'altération. (Cette donnée, nous le verrons ci-après, est conforme aux théories les plus récentes.)

Pour ce qui est de l'altération de la matière grasse, les facteurs d'oxydation seraient, pour WRIGHT [31], le cuivre, l'acidité et la température (en dehors de l'action spécifique de certaines bactéries). Mais de tous ces facteurs, le cuivre serait de beaucoup le plus important surtout en ce qui concerne la rapidité de l'oxydation.

Cette conclusion est confirmée par SAMUELSON [32] qui attache aux sels de cuivre un pouvoir catalytique plus grand qu'aux sels de fer. L'action des sels de fer est loin d'être nulle et chacun sait l'attention qu'il faut porter à la teneur en fer des eaux de lavage du beurre. Samuelson insiste de son côté pour rejeter le fer étamé du 
travail des produits de laiterie acides. Ce même auteur tient le nickel pour bon quand il est très pur, ce qui est rare, et l'aluminium pour excellent s'il titre au moins $99,5 \%$, ce qui est courant actuellement.

Les observations et les expériences de KRENN-[33] apportent également la preuve d'un rapport étroit entre le contact du cuivre et l'apparition de saveurs désagréables dans le lait: "Dans une laiterie, dit-il, les laits bruts et les laits pasteurisés au laboratoire ne prenaient aucun mauvais goût. Seuls, les laits qui passaient dans les appareils de la laiterie prenaient un goût de suif très prononeé 24 heures après la pasteurisation. " Cet auteur est même allé plus loin : il a dosé le cuivre đans de nombreux laits présentant ou ne présentant pas le défaut en question et il a noté que lorsque la teneur en cuivre augmente dans le lait, le mauvais goût apparaît plus vite et est plus intense. Il conclut en disant que les pompes à lait en laiton ou en bronze sont particulièrement dangereuses sous ce rapport.

Nous pourrions multiplier les citations; toutes conduisent à cette conclusion que le cuivre est responsable du goût de suiffage. Nous verrons un peu plus loin comment il faut préciser cette affirmation en montrant que le cuivre à lui seul n'est pas toujours capable d'engendrer le phénomène. Mais nous pouvons, avec tous les auteurs qui se sont occupés de la question, affirmer que le cuivre est un des facteurs fondamentaux de l'apparition d'un des mauvais goûts les plus fréquents dans le lait et ses dérivés.

Il va de soi que si ces observations sont exactes on doit pouvoir reproduire la saveur " huileuse » dans le lait en y introduisant directement des sels de cuivre.

Effectivement KENDE [34] y est parvenu en apportant à du lait sans mauvais goût une trace de sulfate de cuivre. De même aussi DIERnhOFER [35] en utilisant $0 \mathrm{mgr} .03$ de cuivre à l'état de chlorure par litre de lait. Les expériences de CsrszaR [36] comportent les mêmes enseignements.

Nous avons personnellement répété ces expériences, et avons obtenu les mêmes résultats. Nous avons même observé qu'en plongeant une lame de cuivre dans du lait non acide et en portant le tout à $65^{\circ}$ pendant 30 minutes, on développe presque toujours un mauvais goût très identique au goût d'huilage. Ce mauvais goût n'apparaît jamais immédiatement mais seulement après 24 ou 48. heures. Nous croyons intéressant de compléter ces notions par des ehiffres qui constituent un complément de preuve.

b) Le cuivre dans le lait. Notions quantitatives. - La dose normale de cuivre contenue dans le lait n'ayant jamais été mis en contact 
avec ce métal est d'environ $0 \mathrm{mgr}$. 5 par litre. G. Bertrand Supplee, Hess, Belliss, Rice sont d'accord sur ce chiffre.

QUAM et HELLWIG [37] indiquent des teneurs variant entre 0 mgr. 25 et 0 mgr. 45 par litre. Ces mêmes auteurs ont démontré que les laits de chèvre, de vache, de brebis contiennent la même quantité de cuivre et ils ont apporté la preuve que cette teneur est tout à fait indépendante de la teneur en cuivre des aliments.

WeICH et BAUER [38] trouvent $0 \mathrm{mgr} .37$ de cuivre par litre de lait n'ayant jamais séjourné dans des récipients en cuirre. D'autres auteurs donnent des chiffres assez différents des précédents : GRENDEL [39] estime que le lait de vache brut contient environ $0 \mathrm{mgr}$. 10 à 0 mgr. 15 de cuivre par litre, chiffre qui serait confirmé par REMy. En revanche pour ZBINDEN [40] la teneur serait de 0 mgr. 6 à 0 mgr. 9.

De toute manière, la moyenne générale serait voisine des premiers chiffres donnés ci-dessus et on peut admettre que, dans l'ensemble, la teneur du lait brut en cuivre est de quelques dixièmes de milligrammes par litre.

Le lait et ses dérivés traités industriellement voient leur teneur en euivre augmenter au contact des appareils en cuivre.

QUam et Helswig [37] donnent pour le lait pasteurisé $0 \mathrm{mgr}$. 7 de cuivre; pour le lait concentré les teneurs de $1 \mathrm{mgr} .8$ à $2 \mathrm{mgr} .7$ par litre; pour le babeurre le chiffre de $2 \mathrm{mgr}$. 5 environ. W вICH et BAUER ont même trouvé jusqu'à $3 \mathrm{mgr}$. 8 de cuivre par litre dans certains laits fortement suiffeux.

RICE [41] qui a fait une étude très complète de la question montre que le cuivre oxydé cède plus de métal que le cuivre poli, que la présence d'oxygène augmente la dissolution, que le gaz carbonique est sans effet, que la température joue un grand rôle (dissolution plus rapide aux températures de pasteurisation qu'à froid), que le saccharose même à $28 \%$ n'augmente pas la dissolution, que le lait acide ne dissout pas plus de cuivre que le lait neutre (l'acide lactique ne saurait done être mis en cause), que, à la centrifugation, la répartition est proportionnelle à la teneur en eau de chaque portion, que le cuivre ne se concentre pas dans la crème, et que, d'une manière générale, la dissolution du cuivre est proportionnelle aux surfaces et aux temps.

Cet auteur montre encore que le passage sur de l'étain, de lait déjà chargé de cuivre entraîne une diminution du taux de cuivre, ce qui explique que les laits condensés si riches en cuivre au sortir des appareils sont trouvés beaucoup plus pauvres ensuite (dans les boîtes de fer-blanc). De même, à égalité de surface de cuivre, un appareil en cuivre désétamé cède moins de cuivre qu'un appareil en cuivre pur. La présence de l'étain diminue la dissolution du 
cuivre; mais en revanche la présence du cuivre accélère la dissolution de l'étain et cela expliquerait que le désétamage des appareils en cuivre soit plus rapide que le désétamage du fer étamé.

L'influence de la température sur la dissolution du cuivre a été précisée par un beau travail de QUAM [42] dont les conclusions sont les suivantes :

La dissolution croît très vite avec la température, passe par un maximum à $85-90^{\circ}$ et décroît ensuite légèrement.

A $100^{\circ}$, on a une dissolution aussi forte qu'à $65^{\circ}$ (contrairement à ce que pensait RICE).

Puisqu'on est fondé à redouter la dissolution de certains métaux dans le lait, il y aura lieu de prendre des précautions spéciales en ce qui concerne le choix et le mode d'emploi des produits de nettoyage et de désinfection.

De nombreuses études ont été faites à ce sujet. La plupart d'entre elles sont très connues. Mais nous estimons que le point sur lequel il y a lieu d'insister le plus est celui du rinçage des appareils dont il n'est jamais question dans ces études. Pour ce qui est des maurais goûts susceptibles d'être causés par les métaux dissous dans le lait, c'est évidemment moins la quantité dissoute pendant le nettoyage qui importe, que la quantité de sels solubles qui subsistent après ce nettoyage contre les parois des appareils.

La plupart des études quantitatives relatives à la teneur en euivre du lait ont été réalisées en effectuant le titrage du cuivre par voie colorimétrique (méthode classique au xanthate éthylique). D'autres ont été réalisées d'une manière un peu différente (KRENN [33]).

D'intéressantes précisions ont été données par GeBHaRD et Sommer [43] eoncernant le dosage du cuivre dans le lait. Il faut admettre que les divergences dans les chiffres donnés par divers auteurs proviennent de la diversité et surtout de la délicatesse des techniques mises en œuvre.

Il est néanmoins parfaitement acquis que le cuivre est une cause de mauvais goût et il est connu que ce métal (comme d'autres d'ailleurs) est capable de se dissoudre dans le lait.

Le remède à cette situation est donc, à priori, extrêmement facile à prévoir : suppression pure et simple de ce métal dans la construction des appareils de laiterie.

$$
* * *
$$

Toutefois une remarque très importante s'impose maintenant. Tous les auteurs qui ont étudié les saveurs anormales du type " huilage " ou "suiffage» sont unanimes à incriminer le cuivre. Mais il est également aequis avec certitude que le cuivre, si employé dans 
nos industries, ne cause pas toujours de mauvais goût. On assiste fréquemment à la disparition en apparence spontanée du goût d'huilage sans pour cela avoir éliminé les moindres possibilités d'introduction du cuivre dans le lait. De nombreuses usines dont le matériel contient du cuivre n'ont jamais eu à souff́rir d'inconvénients de ce genre.

Donc, si le cuirre paraît bien nécessaire à l'apparition des saveurs. anormales de ce type, il est certain que sa présence n'est nullement suffisanie.

D'autres facteurs certainement interviennent, à côté du cuivre ou en même temps que lui, pour déterminer les saveurs anormales d'huilage ou de suiffage, e'est-à-dire dues à une oxydation de la matière grasse.

Ces considérations vont nous mener tout naturellement à l'étude d'un nouveau groupe de causes, que nous séparons du précédent pour faciliter l'exposé mais qui, en réalité, devrait rester lié à la question du cuivre comme nous allons le voir.

$$
\text { *** }
$$

\section{5 . Etude du cinquième groupe de causes.}

Modifications des éléments normalement contenus dans le lait par des facteurs d'ordre diastasique.

Disons tout de suite qu'il s'agit encore et à peu près exclusivement d'altération de la matière grasse du lait. Nous avons rappelé précédemment qu'il y avait lieu de distinguer deux sortes d'altérations de cette matière grasse : l'hydrolyse (qui mène au rancissement) et qui est presque toujours d'origine diastasique ; l'oxydation (qui mène au suiffage) et qui est à la fois d'origine diastasique et physico-chimique.

Les facteurs d'ordre physicochimique que nous venons d'étudier concernaient done seulement l'oxydation, mais cette étude doit être complétée ici par des considérations biologiques.

Nous étudierons successivement les actions diastasiques oxydantes, puis les actions diastasiques hydrolysantes.

\section{LES OXYDATIONS DIASTASIQUES DU LAIT}

Toute cette importante question se rapporte aux mauvais goûts désignés sous les noms de " huilage ", de "suiffage "; de " goût fade ", de "moisi ", etc. On doit vraisemblablement y rattacher certaines autres saveurs anormales encore moins nettement définies, qui d'ailleurs peuvent résulter de la coexistence de celles-ci avec quelquesunes des autres précédemment étudiées.

Cette question est restée longtemps confuse et sans explication satisfaisante. Nous n'entreprendrons pas d'en faire l'histoire 
détaillée. Nous nous contenterons d'en résumer les étapes et d'en donner la théorie actuelle qui satisfait pleinement à nos besoins. Nous désignerons ce groupe de saveurs par le terme unique d'huilage.

a) La saveur anormale d'huilage est d'origine biologique. - Le fait que la crème pasteurisée à haute température $\left(95^{\circ}\right)$ donne du beurre moins oxydable que la erème crue (toutes choses égales d'ailleurs) montre qu'on a affaire à une action biologique (microbe ou diastase) annihilée par la chaleur.

b) Cette saveur n'est pas le fait de microbes. - MaттісK [30] a bien montré que du lait, normal à la sortie des appareils de pasteurisation, peut acquérir cette saveur anormale après un séjour de 15 à 20 heures à basse température sans que la richesse bactérienre ait augmentẻ.

En isolant (à de nombreuses reprises) les microbes présents dans ces produits présentant un mauvais goût d'huilage, et en les repiquant dans du lait; il a été impossible d'y faire naître ce même mauvais goût.

$\mathrm{Si}$ on rapproche ces deux séries de faits on est fortement tenté d'en déduire que la cause du phénomène est d'origine diastasique. Mais :

c) Des peroxydases naturelles pures ne causent pas cette altération dans du lait pur. - PALMER et ses collaborateurs [44] extraient la peroxydase du radis noir par la méthode de Willstätter et l'introduisent dans de la orème pasteurisée 10 minutes à $82^{\circ}$, ensemencée ensuite à l'abri de l'air à $0^{\circ} \mathrm{ou}$ à $20^{\circ}$. Aucune altération n'a pu être observée dans la suite comparativement à des témoins non additionnés de diastase et cependant la peroxydase est partout présente et active ainsi qu'on a pu le mettre en évidence. Palmer et ses collaborateurs, qui ont fait une étude très serrée de la question et qui pensaient que l'altération suiffeuse devait être due à une oxydase naturelle, concluent que la peroxydase seule n'est pas un facteur suffisant de l'oxydation de la matière grasse du lait et de l'apparition de la saveur anormale étudiée.

d) La peroxydase doit être activée par un métal pour provoquer l'oxydation. - Le cuivre et la diastase oxydante paraissant l'un et l'autre responsables de certaines oxydations, mais étant très souvent incapables de les produire isolément, il a paru logique d'admettre la nécessité de leur présence simultanée. Cette déduction, conforme d'ailleurs aux théories actuelles sur l'action des diastases, cadre parfaitement avec les faits.

C'est à Kende $[45,46]$ que revient le mérite d'avoir pensé le premier à baser sur cette conception l'explication de l'apparition du goût d'huilage ou de suiffage dans le lait et ses dérivés. 
Il va de soi que d'autres facteurs sont également nécessaires : une matière oxydable (doubles liaisons de la matière grasse) et une matière oxydante (l'oxygène). Des expériences directes ont été faites par MatTick [30] qui montrent bien l'absolue nécessité de l'oxygène.

\section{LE MECANISIME DE L'OXYDATION DIASTASIQUE DU LAIT}

Ainsi done, il faut admettre avec KENDE que le mauvais goût d'huilage ne se produira que si on rencontre à la fois dans le lait :

Les corps gras non saturés,

La diastase oxydante,

Le cuivre (à l'état métallique ou à l'état de sel),

Le facteur oxygène, lui, peut être considéré comme toujours présent dans la pratique.

Si un seul de ces facteurs vient à manquer il n'y aurait pas apparition de mauvais goût. Nous verrons d'ailleurs plus loin que, même si ces trois facteurs sont présents simultanément, le phénomène peut ne pas apparaître, mais pour une raison toute différente qui n'a rien à voir avec le mécanisme de l'oxydation proprement dite.

Le mécanisme de l'oxydation conduisant au goût d'huilage se ramènerait donc dans l'état actuel de nos connaissances à ceci : fixation d'oxygène sur les doubles liaisons des acides gras non saturés de la matière grasse du lait sous l'influence d'une diastase oxydante activée par des traces de métaux.

Disons quelques mots de chacun de ces trois facteurs simultanément nécessaires.

a) Les acides gras non saturés. - La matière grasse normale du lait renferme des acides gras à double liaison éthylénique en quantité très variable suivant la nature de l'alimentation; il est acquis que certains aliments (tourteaux oléagineux par exemple) peuvent l'augmenter notablement.

b) La diastase oxydante. - Pour KENDE cette diastase oxydante serait une diastase nouvelle à laquelle il donne le nom d'oléinase. Elle serait détruite vers $80-85^{\circ}$ mais serait respectée par la pasteurisation basse.

Pour KENDE cette diastase ne serait pas liée à la matière grasse du lait, mais à certaines formes de protéines (albumine, globuline). Des protéines pures extraites du lait et introduites dans un petit-lait artificiel exerceraient une action oxydante.

Cette diastase serait toujours présente dans le lait mais en quantités variables suivant l'individu, l'intensité de la lactation, l'allure physiologique ou pathologique de la sécrétion mammaire. 
c) Les traces de cuivre. - La diastase responsable de ces oxydations semble devoir être activée par des traces de métaux, de cuivre en particulier. Une objection peut être faite immédiatement : puisque le lait contient normalement et régulièrement de petites quantités de cuivre ( $1 / 2 \mathrm{mgr}$. par litre) on pourrait supposer que tous les laits doivent présenter toujours ce phénomène d'oxydation et donc toujours le mauvais goût d'huilage.

Il faut bien noter que le cuivre normal du lait n'y est pas contenu à l'état de sels de cuivre libres mais à l'état de complexes organométalliques où il est masqué suivant la théorie classique et incapable de catalyser une réaction d'oxydation. Au contraire, le cuivre réintroduit dans le lait l'est toujours sous forme de sel de euivre dissocié, done d'ions métal libres, e'est-à-dire actifs, capables de jouer leur rôle de catalyseurs d'oxydation.

Telle est done la théorie actuelle de la formation du goût d'huilage. Elle suppose l'existence de trois facteurs simultanés (corps gras non saturés, diastase oxydante, sels de cuivre).

Si cette théorie est exacte, en s'arrangeant pour que ees trois facteurs soient bien présents simultanément on devrait toujours voir apparaître le mauvais goût d'huilage. Or, il n'en est rien. Dans de très nombreux cas où la théorie permet de prévoir le phénomène, celui-ci n'apparaît pas.

Un nouvel aspect de la question doit en effet être envisagé.

\section{L'ACTION EMPECCHANTE DE CERTAINES SUBSTANCES RÉDUCTRICES}

Le phénomène fondamental, cause du goût d'huilage, est, avonsnous dit, une oxydation des graisses d'origine diastasique.

Si le milieu contient des substances réductrices capables de s'opposer à l'oxydation, on peut prévoir que le goût d'huilage ne se produira pas. L'expérience confirme cette prévision.

De même que les laits contiennent en quantité variable des diastases oxydantes, activées ou non, ils contiennent des substances réductrices d'origine alimentaire ou microbienne. La quantité de ces substances et leur structure est très variable. Celles qui nous intéressent ici sont seulement celles qui possèdent une activité naturelle particulière sur la nature de laquelle, d'ailleurs, nous sommes assez mal renseignés.

KENDE a émis à ce sujet une hypothèse séduisante basée sur le raisonnement et les faits suivants : on sait que l'aptitude au "suiffage " est en relation aveo l'alimentation; elle diminue quand l'alimentation est à base de fourrages frais ; elle augmente avec les fourrages secs. On peut done penser que ces substances réductrices inhibitrices de l'oxydation sont contenues dans les aliments. Effectivement des macérations aqueuses, froides, de fourrages frais 
possèdent un pouvoir inhibiteur très marqué vis-à-vis de l'oxydation de la matière grasse du lait auquel on les mélange. Ces substances, d'ailleurs très actives, se rencontreraient donc dans le lait en petite quantité et à dose très variable. On constate en outre qu'elles passent dans le sérum du lait. KENDE a vérifié que les constituants normaux du sérum n'ont pas d'activité à cet égard et il émet l'hypothèse que ces substances inhibitrices ne peuvent guère être assimilées qu'au groupe des diastases ou des vitamines. Comme, d'autre part, à la température à laquelle sont détruites les diastases, ces substances inhibitrices sont encore actives (la pasteurisation ne détruirait pas ce pouvoir réducteur), KENDE pense qu'on a peut-être affaire à une vitamine. Certains faits pourraient la rapprocher de la vitamine C. Effectivement l'acide ascorbinique possède un certain pouvoir de protection vis-à-vis de l'oxydation du lait. Mais d'autres faits montrent qu'il ne faut pas pousser cette assimilation trop loin. Pour KENDE les substances inhibitrices seraient complexes et elles se rapprochent du groupe des vitamines par un certain nombre de leurs propriétés.

Le mécanisme de leur action serait facile à concevoir : comme elles sont plus sensibles à l'action des catalyseurs d'oxydation que la matière grasse elle-même, elles sont attaquées les premières par les facteurs oxydants dont elles annulent l'influence aussi longtemps qu'elles ne sont pas elles-mêmes oxydées.

Il faut noter aussi que des substances jouant un rôle analogue sont sécrétées par de nombreux microbes. D'où une double origine possible de ces substances inhibitrices de l'oxydation : alimentaire ou microbienne.

De nombreux faits concordent parfaitement avec ces conceptions. Citons-en seulement quelques-uns.

(Dans tous les cas il s'agit de laits dont on s'est arrangé pour qu'ils contiennent sûrement les trois facteurs d'oxydation précédemment décrits) :

a) Si ce lait provient de vaches nourries entre autres choses avec du fourrage frais, riche source de substances réductrices, le goût d'huilage n'apparaît pas.

b) Si le foin est sec et vieux (e'est-à-dire oxydé), il entraîne une protection beaucoup moindre contre l'huilage.

c) Si les fourrages frais sont totalement absents (toutes choses égales d'ailleurs), il n'y a que très peu de réductases d'origine alimentaire et le mauvais goût n'est plus empêché. C'est ce qui avait été observé par divers auteurs et en particulier par MAYER [47].

d) $\mathrm{Si}$, les trois facteurs d'oxydation étant toujours présents 
et l'alimentation impropre à protéger le lait, on laisse celui-ci s'infecter fortement, les nombreux microbes qui y prolifèrent et qui sécrètent des substances réductrices, s'opposent à l'apparition du mauvais goût.

e) Si ce même lait oxydable et non protégé, mais infecté, est maintenu au froid (ce qui empêche la pullulation des germes), le mauvais goût apparaît, alors qu'à la température ordinaire où les germes prolifèrent abondamment, le mauvais goût apparaît beaueoup moins ou pas du tout.

Ces données d'une importance capitale expliquent de très nombreux faits, en particulier les suivants : les laits d'hiver sont presque seuls à présenter le mauvais goût parce que l'alimentation ne comporte pas de fourrages frais et parce que les infections microbiennes sont moins vives. En été, pas ou peu de goût d'huilage parce que les vaches consomment de l'herbe et parce que les laits sont généralement plus pollués. Les laits refroidis à basse température sont plus aptes à donner le mauvais goût que les laits moins refroidis parce que les microbes laissés ou réintroduits se développent moins et protègent moins contre l'huilage, dans le premier cas que dans le second. Notons aussi qu'on s'explique mieux maintenant pourquoi ce sont les laits les plus purs qui sont le plus souvent l'objet de ees inconvénients.

Il faut reconnaître une haute valeur à cette théorie qui explique de très nombreux faits importants et du même coup nous met sur la voie des remèdes à envisager.

\section{LES REMĖDE A APPORTER AUX PHÉNOMENES DE L'HUILAGE}

Nous savons que trois facteurs sont simultanément nécessaires à l'apparition de ces saveurs anormales. Nous connaissons également les facteurs empêchants.

Nous devons donc nous ingénier à supprimer au moins l'un des facteurs du premier groupe, ou bien à produire l'un des facteurs d'inhibition du second groupe.

La raison commande évidemment de chercher simultanément à éliminer tous les facteurs du premier groupe et à produire ceux du second groupe. On aura ainsi le maximum de sécurité et de résultats.

\section{A. SUPPRESSION DES FACTEURS D'OXYDATION.}

Nous en connaissons trois : les matières grasses non saturées oxydables, les diastases oxydantes, le cuivre.

Tranchons tout de suite la question des diastases oxydantes qui, faisant partie intégrante de tout lait physiologiquement normal, à des doses variées il est vrai, ne peuvent pas être complètement éliminées. 
Pour ce qui est des acides gras à doubles liaisons, surtout apportés dans la ration par les huiles végétales, nous aboutirions à la condamnation des tourteaux oléagineux. Cette interdiction est impossible dans la pratique. On peut seulement souhaiter de diminuer le plus possible dans la ration l'introduction de graisses végétales non saturées.

Comme d'autre part les industriels du lait ne peuvent pas toujours agir sur l'alimentation des vaches de leurs produeteurs, le seul facteur d'oxydation sur lequel ees industriels peuvent vraiment agir est la suppression totale et rigoureuse du cuivre dans leurs installations.

L'attention doit être portée, d'ailleurs, non seulement sur le cuivre, mais également sur certains autres métaux capables de catalyser, à un degré moindre il est vrai, ces oxydations. Nous n'hésitons pas à dire que, pour le point de vue qui nous occupe, il faudrait s'interdire le cuivre et ses alliages, le cuivre chromé, le cuivre ou le laiton étamé (qui se désétame facilement). Les seuls métaux à retenir pratiquement seraient done les aciers spéciaux et l'aluminium (indépendamment de l'émail bien entendu).

\section{B. Production des Facteurs de réduction.}

Dans le cas où aucun des facteurs d'oxydation ne pourrait être supprimé (et même par prudence dans le cas contraire) il y a lieu d'envisager la production des facteurs du second groupe.

Ils concernent l'alimentation et les réductases bactériennes.

Pour l'alimentation il n'y a qu'une solution : remplacer les aliments pauvres en substances protectrices (betteraves, tourteaux, drèches, pulpes) par des aliments riches en réductases (fourrages frais).

En été, rien de plus simple : Le remède d'ailleurs est automatique, on n'observe pratiquement jamais d'huilage quand les vaches sont aux pâturages.

En hiver il y a impossibilité matérielle à réaliser ees conditions. Tout au plus pourrait-on faire appel au foin; mais la dessiccation lui a fait perdre la majeure partie de son pouvoir protecteur.

Done il n'y a pas de remède à envisager de ce côté (1).

Pour ce qui est des réductases bactériennes on aboutit à ce résultat qu'il faudrait conserver d'assez nombreux microbes dans le lait (et à condition encore de ne pas trop refroidir celui-ci) pour obtenir la

(1) Notons toutefois, à titre documentaire, que le fait d'intrcduire dans la ration des vaches du eharbon animal, à la dose de $10 \mathrm{gr}$. par animal et par jour, fait disparaitre selon MAJER [34] le goût d'huilage après quelques jours de régime. Ce fait qui peut être fort intéressant dans la pratique et qui est capital pour la théorie exposée ici, puisqu'il confirme la nécessité de l'apport de facteurs de réduction, reste dans les possibilités du producteur, et l'industriel ne saurait l'imposer. 
protection désirée. Il est évident que ce remède ne peut pas être davantage retenu,

Toutefois, nous devons ouvrir ici une parenthèse importante.

KENDE, fidèle à sa théorie de l'huilage, s'est demandé si, dans le nombre des bactéries fortement productrices de réductases, il ne s'en trouverait pas qui soient absolument incapables d'altérer le lait. Kertesz, de Budapest, s'est attaqué au problème et l'a résolu. Il est parvenu à isoler une bactérie remplissant ces conditions (Bacterium frigidum neutrale Kertesz) qui ne produit aucune acidité ni aucune altération dans le lait, possède un pouvoir réducteur élevé, et se développe bien à froid.

Cette bactérie se présente comme un coccobacille immobile, non sporogène, gram négatif, souvent groupé par deux ou même en courtes chaînes. Elle pousse bien à $18^{\circ}$, également à froid, mais ne se développe pas à $37^{\circ}$. Elle n'attaque pas les sucres réducteurs, ne donne pas d'indol en peptone, et n'a aucune influence sur la composition, les propriétés et le goût du lait, même après 14 jours de culture. Absolument inoffensive, elle est facilement détruite à $63^{\circ}$ en quelques minutes.

Le microbe de Kertesz présente donc toutes les conditions requises et, fait capital, la réductase qu'il produit est beaucoup plus résistante à la chaleur que le bacille lui-même, de sorte que les cultures tuées présentent la même propriété de protection contre l'huilage que les eultures vivantes, ce qui a permis la préparation industrielle et la vente de cultures sèches et actives.

On a ainsi le moyen de "vacciner " le lait (selon l'expression de Kertesz) contre le mauvais gout de l'huilage en l'additionnant de réductase.

Nous avons pu nous procurer des cultures sèches de Bact. frigidum neutrale et nous les avons expérimentées :

1. Ayant mis un lait dans la situation de donner un goût d'huilage (et l'ayant finalement obtenu sur un témoin), nous avons traité ce lait par la méthode Kertesz et nous avons parfaitement empêché l'apparition du goût d'huilage même en présence de fortes doses de sels de cuivre.

2. D'autres expériences ont été conduites de la façon suivante : du lait additionné de cultures sèches a été pasteurisé 30 minutes à $65^{\circ}$ en présence de cuivre. Ce lait n'a pas présenté le goût d'huilage alors que le même lait, traité de la même façon, sans addition de cultures sèches, l'a présenté à un haut degré.

3. La dose de culture sèche nécessaire à l'obtention d'un résultat positif est d'environ $4 \mathrm{gr}$. pour 100 litres de lait.

Notons d'ailleurs que les cultures dont il s'agit sont des cultures 
enrichies par Kentesz par ensemencements successifs dans le même milieu, entrecoupés de pasteurisations basses. Le microbe était ainsi détruit après avoir produit la diastase, celle-ci étant toujours respectée.

La nécessité de cette quantité élevée de cultures déjà enrichies est, à notre avis, une objection d'ordre pratique assez sérieuse. I1 faudra pour traiter 10.000 litres de lait aumoins $400 \mathrm{gr}$. d'une culture sèche enrichie, dont le prix est loin d'être négligeable (environ 200 francs le kilo).

Les essais que nous avons entrepris ont done parfaitement répondu à ce qu'on pouvait en attendre au point de vue technique.

Nous croyons toutefois devoir signaler que l'usage de ferments en poudre de Kertesz pourrait soulever diverses objections soit dans l'ordre de l'hygiène (si des garanties formelles n'étaient pas données et tenues), soit dans l'ordre de la répression des fraudes (la législation française s'opposerait à l'adoption de cette technique dans l'état actuel de la "définition" du lait).

C. Résumé des Remèdes a APPoRter a "L'HUilage 》.

L'apparition de mauvais goût d'huilage est done, finalement, sous la dépendance de 5 facteurs : 3 facteurs d'oxydation dont la présence simultanée tend à produire le phénomène, 2 facteurs de réduction qui peuvent l'un ou l'autre s'opposer à son apparition.

En ce qui concerne les premiers (acides gras à doubles liaisons dans l'alimentation, diastases oxydantes du lait, cuivre dans le matériel), un seul - la présence du cuivre - est à la portée de l'industriel.

En ce qui concerne le second, seul, l'usage de ferments de Kertesz à mélanger au lait dès la traite ou, tout au moins, avant la pasteurisation, peut être envisagé au point de vue technique.

$\mathrm{Ce}$ dernier remède, certainement efficace, est-il absolument acceptable à tous les points de vue ? Peut-il être employé sans aucun inconvénient hygiénique, légal, ou autre? De nouvelles études méritent d'être entreprises.

En tout cas il semble que la suppression absolue du cuivre, malgré les problèmes qu'elle soulève, puisse et même doive être envisagée dans la construction du matériel de laiterie (cuivre, alliages de cuivre, cuivre étamé, etc.). 